\title{
The rheology of concentrated suspensions of arbitrarily-shaped particles
}

\author{
I. Santamaría-Holek ${ }^{1}$ and Carlos I. Mendoza ${ }^{2}$ \\ ${ }^{1}$ Unidad Multidisciplinaria de Docencia e Investigación, Facultad de Ciencias Ju- \\ riquilla, Universidad Nacional Autónoma de México, Boulevard Juriquilla 3001, \\ Querétaro, 76230, Mexico \\ ${ }^{2}$ Instituto de Investigaciones en Materiales, Universidad Nacional Autónoma de \\ México, Apdo. Postal 70-360, 04510 México, D.F., Mexico
}

\section{Introduction}

When particles are suspended in an homogeneous isotropic fluid, the viscosity of the resulting complex fluid is increased. In the case of dilute suspensions, the increase in viscosity as a function of the volume fraction $\phi$ was firstly determined for spherically shaped particles by Einstein in 1911, [1]. Some years later, extensions of Einstein's work appeared and new formulas for the viscosity as a function of the volume fraction for solid ellipsoidal particles and emulsions were also derived by Jeffery (1922) and Taylor (1932), respectively [2,3].

For sufficiently low particle concentrations, the viscosity $\eta$ of a suspension can in general, be written as,

$$
\eta(\phi)=\eta_{0}\left(1+[\eta] \phi+k_{H} \phi^{2}+\ldots\right),
$$

where $\eta_{0}$ is the solvent viscosity, $[\eta]$ is the low filling fraction intrinsic viscosity, $k_{H}$ is the so called Huggins coefficient, and $\phi$ is the volume fraction of the particles. The value of $[\eta]$ depends on the particle shape. Although its calculation is difficult, there are few cases where $[\eta]$ has been obtained analytically as in the case 
of spheres [1], ellipsoids [4], 5], long cylinders [6] and dumbbells consisting of two identical spheres [6], [7]. Fortunately, accurate numerical approaches have been developed to calculate the intrinsic viscosity of arbitrarily shaped particles [8], [9].

The large amount of work devoted to determine the relation between viscosity and concentration under different physical conditions that reflect experimental protocols [10,11,12,13], is clearly connected with the important role that suspensions and emulsions play in almost all fields of industry, medicine and biologyrelated soft-matter systems.

Many remarkable theoretical and, more recently, numerical works have made central contributions to the understanding of the rheology of concentrated suspensions (see for instance, Refs. [14][29]). Essentially, most of these works introduce particle correlations by taking into account hydrodynamic interactions and provide a conceptual framework that explains how the microstructure of the systems modifies the behavior of the viscosity as a function of the volume fraction. However, the quantitative description of the problem still constitutes an open challenge for theoretical descriptions even in the simplest case of spherical particles.

Several models have been proposed in order to extend the range of applicability of Einstein's expression to larger volume fractions [10]. Semi-empirical [33,30] and effective-medium [34]-[37] models intended to extend the quantitative description of the experimental dependence of the effective viscosity of hard particle suspensions at arbitrary volume fractions have been proposed long ago. Among them we can mention the one derived by Saito [14] that accounts for hydrodynamic interactions between uncorrelated particles

$$
\eta(\phi)=\eta_{0}\left[1+[\eta]\left(\frac{\phi}{1-\phi}\right)\right]
$$


Other approaches include the use of differential effective-medium theories (DEMT). Krieger and Dougherty [36] derived the following expression that incorporates excluded volume using a crowding effect introduced by Mooney [38]

$$
\eta(\phi)=\eta_{0}\left(1-\frac{\phi}{\phi_{\max }}\right)^{-[\eta] \phi_{\max }},
$$

with $\phi_{\max }$ the filling fraction at maximum packing. This formula agrees reasonably well with the experimental data particularly in the low concentration regime. Moreover, this relation reduces to the correct Einstein's equation in the limit of infinite dilution. Very recently, Bullard and coworkers [42] also used DEMT techniques to derive a similar relationship for suspensions of particles that may themselves incorporate some of the solvent either by solvation or by occlusion in interstitial pores. In the case of spheres their expression adopts the form

$$
\eta(\phi)=\eta_{0}(1-K \phi)^{-[\eta] / K},
$$

where the factor $K$ considers flocculation of the particles thus representing the ratio of the volume of the clusters to the volume of the particles forming the clusters [42].

The original formulations of DEMT were based on Einstein's expression, providing moderate agreement with experimental results. It is important to stress that one common characteristic of these models is that they describe acceptably well the viscosityconcentration relation at low concentrations but fail to describe correctly the whole concentration regime. As we will explain later, this is because these theories do not incorporate appropriately the correlations introduced by the excluded volume effects.

A different approach to calculate the viscosity of suspensions is based on the expected theoretical divergence of viscosity near the percolation threshold [31]

$$
\eta(\phi) \sim \eta_{0}\left(1-\frac{\phi}{\phi_{c}}\right)^{-2},
$$


which, according to Douglas and coworkers [32, should be independent of the shape of the particles in suspension.

Bicerano et al. [30] examined the viscosity of suspensions of different hard bodies, and proposed a formula for the relative viscosity that provides a smooth transition between the dilute and the concentrated, Eq.(5), regimes and is valid for low-shear

$$
\eta(\phi)=\eta_{0}\left(1-\frac{\phi}{\phi_{c}}\right)^{-2}\left[1+C_{1}\left(\frac{\phi}{\phi_{c}}\right)+C_{2}\left(\frac{\phi}{\phi_{c}}\right)^{2}\right],
$$

with

$$
C_{1}=[\eta] \phi_{c}-2
$$

and

$$
C_{2}=k_{H} \phi_{c}^{2}-2[\eta] \phi_{c}+1 .
$$

This theoretical formula accounts for both the low density exact results from hydrodynamics as well as the large volume fraction semi-empirical expansions. Pryamitsyn and Ganesan [43] proposed to extend the domain of validity of this expression to arbitrary shear rates by considering the viscosity percolation threshold $\phi^{*}(P e)$ a function of the Peclet number.

Recently, a model has been proposed that introduces in appropriate form the correlations introduced by the excluded volume effects and gives an excellent quantitative description of the viscosity of solid and liquid suspensions of spherical particles at arbitrary filling fractions Ref. [39,40]. This model incorporates an effective filling fraction $\phi_{\text {eff }}$ that leads to an universal representation of all experimental results in a master curve that suggest the role of $\phi_{\text {eff }}$ as a scaling variable for the viscosity of these systems.

Despite the importance of the theoretical and numerical analysis performed mostly in the case of spherical particles, in real systems the assumption of sphericity of the suspended particles is not always satisfied since polydispersity and different particle shapes have to be taken into account [41]. Thus, the main objective of this article is to propose a continuum-medium description for the 
viscosity-concentration relation for different particle shapes as long as they are not too elongated. The description is derived using DEMT techniques introducing correlations between particles through an effective volume fraction that incorporates excluded volume effects. These effects are responsible for the scaling properties of the suspensions and show to be universal independently of the shear rate and the shape of the particles.

The article is organized as follows, in Section II we propose a first correction to Eq. (1) that takes into account excluded volume effects and use it as the starting point of a differential effective medium approach. In Section III we compare the predictions of our model with previous theories and with various experimental results for different particle shapes. Finally Section IV is devoted to conclusions.

\section{Correlations and DEMT approach}

The main difficulty when dealing with suspensions at large concentrations is to take into account the correlations among particles. Although many efforts have been devoted to microscopically calculate the viscosity of a suspension of hard particles taking into account the hydrodynamic interactions, the enormous mathematical complications associated to the many body problem only permit to find corrections applicable to the low concentration regime. However, in first approximation, such correlations can be considered as follows: the contribution by the particles to the total stress tensor $\overline{\boldsymbol{\Pi}}_{p}^{V}$ of the suspension is given in terms of an average over the volume $V$ of the system in the form

$$
\bar{\Pi}_{p}^{V} \simeq \frac{N}{V} \int \boldsymbol{\Pi}_{p}^{(1)} d V
$$

where we represented the single particle contribution to the stress tensor by $\Pi_{p}^{(1)}$. The upper $V$ in Eq. (9) stands for the volume average and the factor $N$ accounts for the contribution of the $N$ 
independent particles. However, as defined in Eq.(9), the average is strictly valid only for a system of point particles [39].

Considering that each particle has a volume $V_{p}$, then the volume average must be performed over the free volume accessible to the particles defined by: $V_{\text {free }}=V-c N V_{p}$. Here $c$ is a geometric factor that takes into account the fact that the complete free volume cannot be filled with particles. Note that, for different shapes of the suspended particles, the value of the constant $c$ will be different. It also contains information about the maximum packing of particles the system may allocate and if the excluded volume effects are taken into account, the suspended phase contribution to the stress tensor is now given by [39], [40]

$$
\begin{aligned}
\bar{\Pi}_{p}^{V_{\text {free }}} & \simeq \frac{N}{V-c N V_{p}} \int \boldsymbol{\Pi}_{p}^{(1)} d V \\
& =\frac{1}{1-c \phi}[\eta] \phi{\overline{\nabla v_{0}^{0}}}^{V}
\end{aligned}
$$

where $\phi=N V_{p} / V,{\overline{\nabla v_{0}^{0}}}^{V}$ is the volume average of the traceless velocity gradient with $v_{0}$ the velocity field of the background fluid [50], [39]. For finite-sized particles, this relation leads to the result that Einstein's expression scales with the excluded volume factor $\phi /(1-c \phi)$ instead of $\phi$, and thus gives the following expression for the viscosity of a suspension

$$
\eta(\phi)=\eta_{0}\left(1+[\eta] \phi_{e f f}\right)
$$

where the effective filling fraction $\phi_{\text {eff }}$ is defined by

$$
\phi_{e f f}=\frac{\phi}{1-c \phi} .
$$

The constant $c$ depends on the filling fraction $\phi_{c}$ which is the critical concentration at which the suspension loses its fluidity. This viscosity percolation threshold generally is greater than the purely geometrical percolation threshold of the particles, but less than or equal to the maximum packing fraction $\phi_{\max }[42]$ and is 
given by

$$
c=\frac{1-\phi_{c}}{\phi_{c}}
$$

The effective filling fraction (12) approaches the bare $\phi$ at low concentrations and becomes 1 at the divergence of the viscosity which occurs at $\phi_{c}$. The fact that the particles can not occupy all the volume of the sample due to geometrical restrictions is taken into account in the crowding factor $c$. For example, for a face centered cubic (FCC) arrangement of identical spheres, the maximum volume that the spheres may occupy is larger than for a random arrangement of spheres.

The formula for the effective viscosity of the suspensions given by relation Eq. (11) can also be written as

$$
\eta(\phi)=\eta_{0}\left[1+[\eta]\left(\frac{\phi}{1-c \phi}\right)\right]
$$

Although in this expression it is clear that $\eta(\phi)$ incorporates the excluded volume corrections for the viscosity of a suspension, hydrodynamic interactions are ignored and therefore one expects its validity been restricted to low concentrations. To improve it, further corrections must appear due to the interactions between particles. In the system under consideration, these interactions are the hydrodynamic interactions which become increasingly important when increasing the filling fraction. The mentioned correlations can be accounted for by using DEMT techniques [42]. This theoretical method is based on a progressive addition of spheres to the sample in which the new particles interact in an effective way with those added in previous stages [10].

Taking Eq. (11) as the starting point, suppose that we increase by $\delta \phi_{e f f}$ the particle concentration in the suspension of viscosity $\eta\left(\phi_{\text {eff }}\right)$ by adding a small quantity $\Delta \phi_{\text {eff }}$ of few new particles. If we treat the suspension into which we add these particles as a homogeneous effective medium of viscosity $\eta\left(\phi_{\text {eff }}\right)$, then the 
new viscosity can be written as

$$
\eta\left(\phi_{e f f}+\delta \phi_{e f f}\right)=\eta\left(\phi_{e f f}\right)\left(1+[\eta] \Delta \phi_{e f f}\right) .
$$

Note that the increase in the effective particle concentration $\delta \phi_{\text {eff }}$ is different from the effective concentration of new particles added at a given stage $\Delta \phi_{\text {eff }}$. This is due to the fact that one has to remove part of the effective medium, which already contains some particles, in order to allocate the new particles. From this, it follows that the fraction of particles of the new effective medium is given by $\phi_{e f f}+\delta \phi_{e f f}=\phi_{e f f}\left(1-\Delta \phi_{e f f}\right)+\Delta \phi_{e f f}$, from which we find

$$
\Delta \phi_{e f f}=\frac{\delta \phi_{e f f}}{1-\phi_{e f f}}
$$

Substituting Eq.16) in Eq.(15) and integrating we finally obtain

$$
\eta(\phi)=\eta_{0}\left(1-\phi_{e f f}\right)^{-[\eta]},
$$

or, using the definition of $\phi_{e f f}$

$$
\eta(\phi)=\eta_{0}\left[1-\left(\frac{\phi}{1-c \phi}\right)\right]^{-[\eta]}
$$

This relation for the effective viscosity of a suspension of rigid particles constitutes a powerful improved generalization of previous theoretical results and empirical proposals, as we will show in the following section. In the limit of low concentrations Eq. (18) always reduces to Eq.(1) and the predicted Huggings coefficient, obtained by expanding Eq. (18) in a virial series, is given by

$$
k_{H}=\frac{1}{2}[\eta]\left([\eta]+\frac{2}{\phi_{c}}-1\right),
$$

that has the form suggested by Douglas (see Eq.(17) of Ref.[42]).

Although the procedure that leads to Eq. (18) is similar to other DEMT [34]-[37], [42], the introduction of excluded volume correlations through the effective filling fraction $\phi_{\text {eff }}$ defined in Eq. 12 and then its role as integration variable in the differential procedure improves remarkably the agreement with experimental data 
when compared to other models, this is shown in Ref.[39] for the special case of hard spheres. This agreement is due to the fact that the use of $\phi_{\text {eff }}$ as integration variable implicitly considers correlations between spheres of the same recursive stage in contrast to other models.

It will be shown in the next sections that our model gives excellent quantitative results at the whole concentration range for different particle shapes. However, at this point it is interesting to compare the functional form of our expression (18) with the one by Bicerano and coworkers, Eq.(6). Their equation was designed explicitly to provide a smooth transition between the semidilute and concentrated regimes. Equation (18) can be written in a form similar to Bicerano's expression to obtain

$\frac{\eta(\phi)}{\eta_{0}}=\left(1-\frac{\phi}{\phi_{c}}\right)^{-2}\left[1+C_{1}\left(\frac{\phi}{\phi_{c}}\right)+C_{2}\left(\frac{\phi}{\phi_{c}}\right)^{2}+C_{3}\left(\frac{\phi}{\phi_{c}}\right)^{3}+O\left(\phi^{4}\right)\right]$.

Here, $\phi_{c}$ can be considered a function of the Péclet number and therefore is no restricted to the low-shear regime. The constants $C_{1}$ and $C_{2}$ are identical to the ones corresponding to the model of Bicerano and coworkers given by Eqs. (7) and (8), and the coefficient of the cubic term is given by

$$
C_{3}=\frac{\phi_{c}^{3}}{6}[\eta]\left([\eta]^{2}-3[\eta]+2\right) .
$$

It is not surprising that the coefficients of the linear and quadratic terms are the same in both models since by construction they reduce to Eq.(1) at low concentrations. Note however that in practice, when using the model of Bicerano and coworkers, Eq.(6), once $[\eta]$ is known for a given particle shape, $\phi_{c}$ and $k_{H}$ are treated as two independent fitting parameters when comparing to experimental data [42]. In our model, on the other hand, $\phi_{c}$ and $k_{H}$ are related by means of Eq. (19), therefore leading to one fitting parameter only. It is important to point out that although similar in form, expression (20) has not the same divergence near $\phi_{c}$ as (6) since the expression in square brackets in Eq. 20 diverges as 
$\phi \rightarrow \phi_{c}$. However, if we make the expansion

$$
\eta(\phi) / \eta_{0}=\left(1-\frac{\phi}{\phi_{c}}\right)^{-[\eta]}\left[1+C_{1}^{\prime}\left(\frac{\phi}{\phi_{c}}\right)+C_{2}^{\prime}\left(\frac{\phi}{\phi_{c}}\right)^{2}+O\left(\phi^{3}\right)\right],
$$

where

$$
C_{1}^{\prime}=[\eta] \phi_{c}-[\eta]
$$

and

$$
C_{2}^{\prime}=k_{H} \phi_{c}^{2}-2[\eta] \phi_{c}\left([\eta]-\frac{1}{2}\right)+[\eta]([\eta]-1),
$$

then, the expression in square brackets converges. This is an important result meaning that the viscosity diverges near $\phi_{c}$ with an scaling that depends on $[\eta]$ and therefore is not universal but depends on the particle shape and shear rate.

\section{Illustrative calculations}

\subsection{Spheres}

The first illustrative example of the procedure is a system of hard spheres which corresponds to take the value $[\eta]=5 / 2$ in the above expressions which leads to the well known Einstein's result, valid for very low concentrations

$$
\eta(\phi)=\eta_{0}\left(1+\frac{5}{2} \phi\right)
$$

Additionally to the models presented in the previous section, other phenomenological formulas have been proposed in order to fit experiments in the largest possible range of volume fractions. For example, Clercx and Schram obtained the following expression for the high-frequency effective viscosity [45]

$$
\eta_{\infty}(\phi)=\eta_{0}\left[1+\frac{\frac{5}{2} \phi+1.42 \phi^{2}}{1-1.42 \phi}\right]
$$

by considering two-particle hydrodynamic interactions only. 

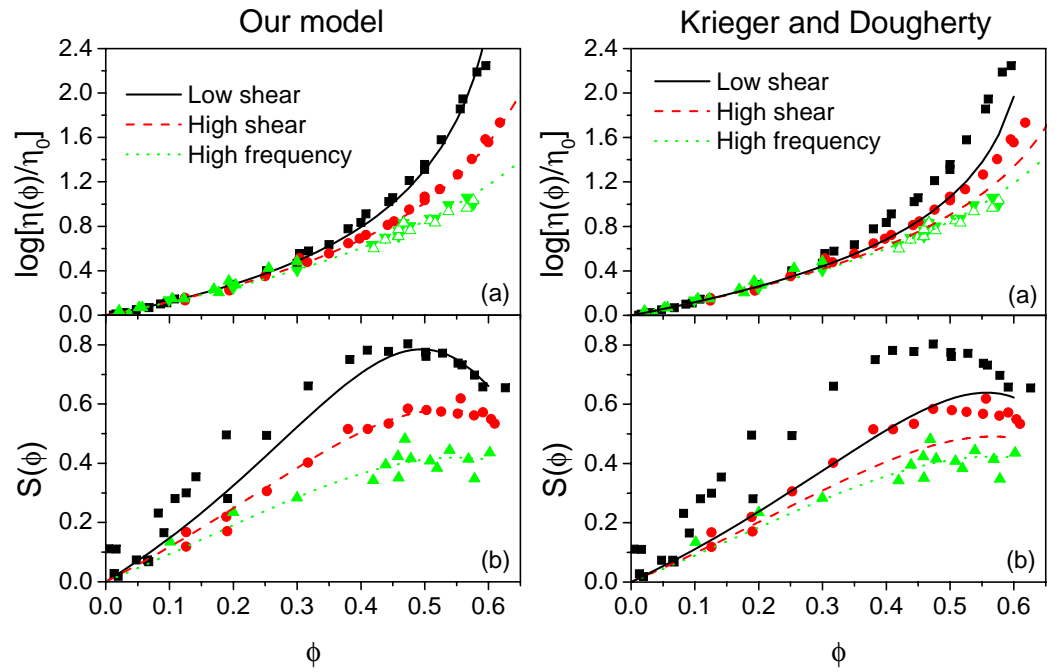

Fig. 1. Left panels: (a) Relative viscosity $\eta(\phi) / \eta_{0}$ as predicted by our model [Eq. 18] ] for spheres $([\eta]=5 / 2)$ at low- and high-shear rates as well as high-frequency as a function of the volume fraction $\phi$. The lines correspond to the predictions of our model with RCP $\phi_{c}=0.63$ (upper line), close packing at FCC $\phi_{c}=0.7404$ (middle line), and fitting parameter $\phi_{c}=0.8678$ (lower line). The measured data are from Refs. 46] (circles) and [47] (triangles). Squares, Zhu et al. [49]; circles, van der Werff et al. [48; triangles, Cichocki and Felderhof [20]. (b) Representation of various viscosity data as suggested by Bedeaux (Ref. [23]). Squares, SJ18 low-shear limit (Refs. [1] and [46]); circles, SJ18 high-shear limit (Refs. [26] and [46]); triangles, high-frequency limit of the real part of the complex shear viscosity (Ref.[48]). The lines are the results of our model with $\phi_{c}=0.63$ (upper line), $\phi_{c}=0.7404$ (medium line), and $\phi_{c}=0.8678$ (lower line). Right panels: The same as the left panels but the lines correspond to the model by Krieger and Dougherty [Eq.(3)].

In order to carry out comparisons of our model with experiments and other models it is important to notice that the value of $\phi_{c}$ in Eq.(13) is a free parameter of the theory to be chosen in order to best fit the experimental results. Nonetheless, this parameter can be chosen beforehand based on physical arguments, and then used to compare with specific experimental situations.

This is done in the left panel of Fig. 1, where the behavior of the relative viscosity $\eta(\phi) / \eta_{0}$ (Fig. 1a, left) is compared with experimental results of de Kruif et al. [46] and of Krieger [47] for low 
and high-shear rates, and with the results of van der Werff et al. [48] and by Zhu et al. [49] at high frequencies as a function of the volume fraction $\phi$. We also plot the values obtained by Cichocki and Felderhof [20] for the high-frequency case. The upper curve represents the prediction of Eq. (18) with $\phi_{c}=0.637$, which corresponds to the random close packing $(\mathrm{RCP})$ of identical spheres. The comparison with the experimental results for low-shear rates is excellent. The middle curve is the prediction of Eq.(18) with $\phi_{c}=0.7404$, which corresponds to FCC close packing. This gives again an excellent agreement with the experimental results for the case of high-shear rates. These results are consistent with the known fact that for low-shear rates the spheres remain disordered while at high-shear rates the equilibrium microstructure of the dispersion is completely destroyed and the spheres adopt an ordered FCC configuration. The lower curve is the prediction of Eq. 18) with $\phi_{c}=0.8678$, which gives again an excellent fit with the infinite-frequency data and with the values obtained by Cichocki and Felderhof [20].

In order to account for thermodynamic interactions between the spheres, Bedeaux proposed the following expression for the viscosity [23]-[24]

$$
\frac{\eta(\phi) / \eta_{0}-1}{\eta(\phi) / \eta_{0}+\frac{3}{2}}=\phi(1+S(\phi))
$$

where $S(\phi)$ is an unknown function of the volume fraction giving the modification of the moment of the friction forces on the surface of a single sphere due to the ensemble-averaged hydrodynamic interactions with the other spheres [24].

As discussed by Bedeaux [23], $S(\phi)$ is a more sensitive representation of the relative viscosity data since its expansion in powers of $\phi$ converges much better. For this reason, the differences between the predictions of the different models are more noticeable in this representation. In Fig. 1b, left panel, we plot the function $S(\phi)$ for various experimental results obtained in a variety of experimental situations and compare with the values obtained with 

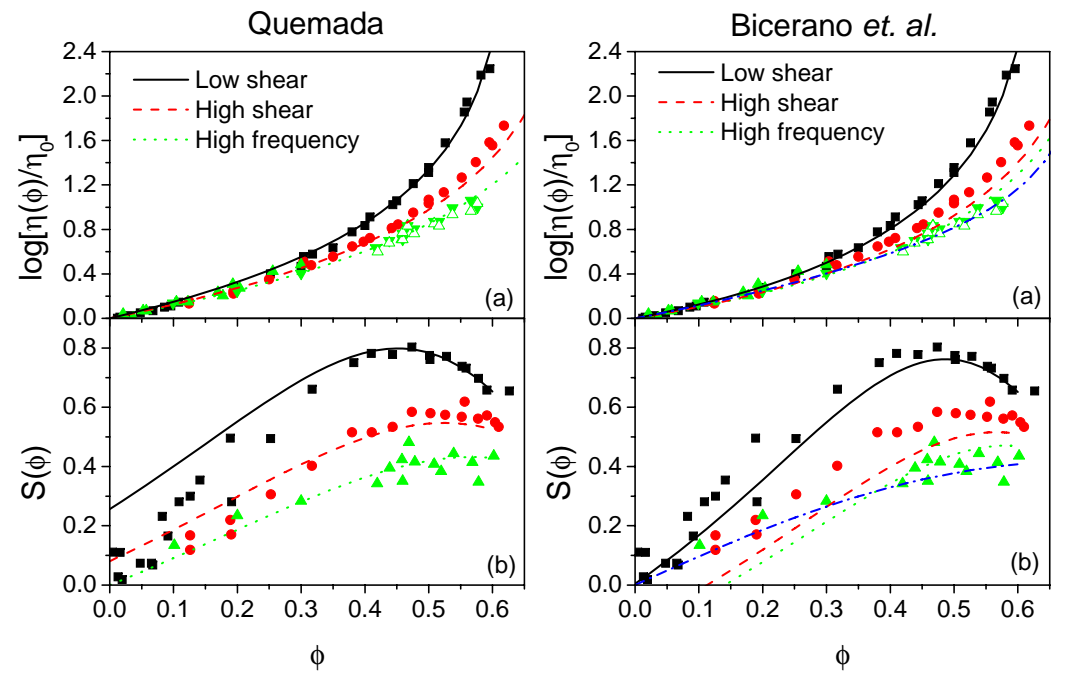

Fig. 2. Left panels: The same as in Fig.1 but the lines correspond to the model by Quemada [Eq.(5)]. Right panels: The same as in Fig. 1 but the lines correspond to the model by Bicerano and collaborators [Eq.(6)]. We also show the high-frequency result by Clercx [Eq. 26)]

(dash-dotted line).

our model. We take the same values of $\phi_{c}$ used previously for the low-shear, high-shear, and high-frequency cases. The agreement with the experimental data is very good especially at large values of $\phi$. Note that for small values of $\phi$ the experimental accuracy of $S(\phi)$ is unsatisfactory which explains the large scatter in the experimental points [23].

As a comparative, in the right panel of Fig. 1 and in Fig. 2 we show the predictions obtained with the models of Krieger and Dougherty [Eq.(3)], Quemada [Eq.(5)], Bicerano [Eq.(6)], and Clercx [Eq. 26)]. In each case, the symbols (a) and (b) state for the direct comparison with experiments (a) and after using Bedeaux's expression (b). It is clear that our model matches the data at all volume fractions better than the other models tested.

Let us stress here that the improvement of our model given by Eq.(18) to the models given by Eqs.(3) and (4) is due to 
the use of $\phi_{\text {eff }}$ given by Eq.(12). Indeed, the model of Krieger and Dougherty can be obtained from the dilute-limit expression $\eta(\phi)=\eta_{0}(1+[\eta] \phi)$ by introducing the effective filling fraction $\phi_{\text {eff }}^{K D} \equiv \phi / \phi_{\max }$, which is larger than $\phi$. This definition of $\phi_{\text {eff }}^{K D}$ underestimates the available volume for the particles at low $\phi$ (and therefore, overestimates $\phi_{\text {eff }}$ ) while tends to the correct limit at high $\phi$. In order to obtain the correct dilute limit, the overestimation of $\phi_{e f f}^{K D}$, has to be compensated by decreasing the hydrodynamic drag factor by the same constant factor $\phi_{\max }$, that is, $[\eta]^{K D} \equiv[\eta] \phi_{\max }$. Then, the dilute-limit expression can be rewritten as

$$
\eta_{r}(\phi)=\left(1+[\eta]^{K D} \phi_{e f f}^{K D}\right)
$$

Now, Eq. (3) can be derived from (28) by following the differential method used in the previous section with the effective filling fraction $\phi_{e f f}^{K D}$ instead of $\phi_{\text {eff }}$ and the corresponding $[\eta]^{K D}$ instead of $[\eta]$. The relative difference $\left(\phi_{e f f}^{K D}-\phi_{e f f}\right) / \phi_{e f f}$ is a decreasing function of $\phi$ that vanishes at $\phi_{\max }$ (we assumed that $\phi_{c}=\phi_{\max }$ in this analysis. Therefore, the overestimation of the filling fraction in $\phi_{e f f}^{K D}$, is progressively less important with increasing $\phi$ and the constant underestimation of the hydrodynamic drag term in Krieger and Dougherty's model cannot be compensated by $\phi_{e f f}^{K D}$. Thus, Krieger and Dougherty's model underestimates the viscosity of the suspension at large volume fractions, as confirmed in Fig. 1b. The same reasoning can be applied to Bullard's model since $1 / K \equiv \phi_{c}$ plays the role of a critical concentration and there is no mathematical distinction between Eq. (4) and the Krieger and Dougherty equation.

In what follows, we show a direct comparison of the functional forms of our proposal and the other models as well as a virial expansion of them. For example, at high-frequencies, a virial expansion of Clercx and Schram's expression, Eq.(26) gives

$\eta_{\infty}(\phi) / \eta_{0}=1+\frac{\frac{5}{2} \phi+1.42 \phi^{2}}{1-1.42 \phi}=1+\frac{5}{2} \phi+4.97 \phi^{2}+7.06 \phi^{3}+O\left(\phi^{4}\right)$,

while our expression, Eq.(18) with $[\eta]=5 / 2$ and $\phi_{c}=0.8678$, 
can be expressed using a Padé approximation as

$\eta_{\infty}(\phi) / \eta_{0}=1+\frac{\frac{5}{2} \phi+0.575 \phi^{2}}{1-1.67 \phi}=1+\frac{5}{2} \phi+4.76 \phi^{2}+7.95 \phi^{3}+O\left(\phi^{4}\right)$,

showing that the second and third virial coefficients are very close. Similarly, the expression by Bicerano and coworkers, Eq. (6), can be written in the case or hard-spheres with $\phi_{c}=0.637$ as

$$
\begin{aligned}
\eta(\phi) / \eta_{0} & =\left(1-\frac{\phi}{0.637}\right)^{-2}\left[1-0.628 \phi+0.84 \phi^{2}\right] \\
& =1+\frac{5}{2} \phi+6.26 \phi^{2}+13.47 \phi^{3}+O\left(\phi^{4}\right)
\end{aligned}
$$

while our expression gives for the same value of $\phi_{c}$

$$
\begin{aligned}
\eta(\phi) / \eta_{0} & =\left(1-\frac{\phi}{0.637}\right)^{-2}\left[1-0.64 \phi+0.415 \phi^{2}\right] \\
& =1+\frac{5}{2} \phi+5.8 \phi^{2}+12.36 \phi^{3}+O\left(\phi^{4}\right) .
\end{aligned}
$$

Both expressions are very similar up to third order in $\phi$. However, as explained before, near $\phi_{c}$ both models predict different asymptotic relations. While Bicerano's model predicts a divergence of the viscosity as $\left(1-\phi / \phi_{c}\right)^{-2}$, our model predicts that the viscosity diverges as $\left(1-\phi / \phi_{c}\right)^{-2.5}$, Eq. 22 , for the case of spherical particles. Our prediction is in close agreement with that of Cheng et. al. [44], that adapted mode coupling theories developed for supercooled liquids and the liquid-glass transition for molecular systems to calculate the low-shear viscosity of colloidal dispersions. Taking into account hydrodynamic interactions, they found that near the glass transition $\phi_{g}=0.62$, the viscosity diverges approximately as $\left(1-\phi / \phi_{g}\right)^{-2.59}$ which is in close agreement with our model if $\phi_{c} \simeq \phi_{g}$.

The proposal by Pryamitsyn and Ganesan[43] to extend Bicerano's expression to arbitrary shear rates by considering the packing fraction $\phi_{c}(\mathrm{Pe})$ a function of the Peclet number can also be tested by expanding in series of $\phi$ for a large shear rate, which means to take $\phi_{c}=0.7404$. In this case Pryamitsyn and Ganesan 

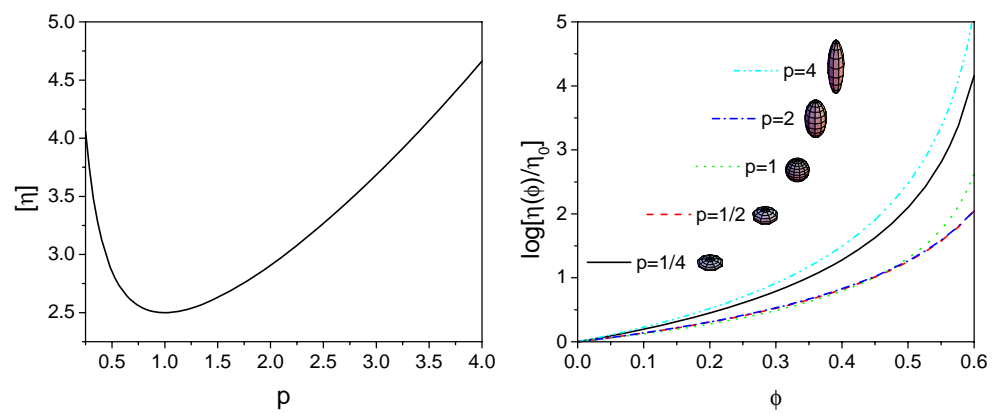

Fig. 3. Left panel: Intrinsic viscosity for ellipsoids as a function of the aspect ratio $p=b / a$. Right panel: Relative viscosity $\eta(\phi) / \eta_{0}$ as predicted by our model for ellipsoids with different values of $p$. The critical packings were assumed to coincide with the MRJ states as obtained in Refs. ([52]) and ([53]): for $p=1 / 4, \phi_{c} \simeq 0.64$; for $p=1 / 2, \phi_{c} \simeq 0.7$; for $p=1, \phi_{c} \simeq 0.637$; for $p=2, \phi_{c} \simeq 0.704$; and for $p=4$, $\phi_{c} \simeq 0.632$.

obtain

$$
\begin{aligned}
\eta(\phi) / \eta_{0} & =\left(1-\frac{\phi}{0.7404}\right)^{-2}\left[1-0.54 \phi+0.622 \phi^{2}\right] \\
& =1+1.6 \phi+3.1 \phi^{2}+5.46 \phi^{3}+O\left(\phi^{4}\right)
\end{aligned}
$$

while our model gives

$$
\begin{aligned}
\eta(\phi) / \eta_{0} & =\left(1-\frac{\phi}{0.7404}\right)^{-2}\left[1-0.201 \phi+0.322 \phi^{2}\right] \\
& =1+\frac{5}{2} \phi+5.25 \phi^{2}+9.94 \phi^{3}+O\left(\phi^{4}\right) .
\end{aligned}
$$

Notice that the agreement is not very good even at first order in $\phi$ where the extended Bicerano's expression do not agree with Einstein's expression for low $\phi$. 

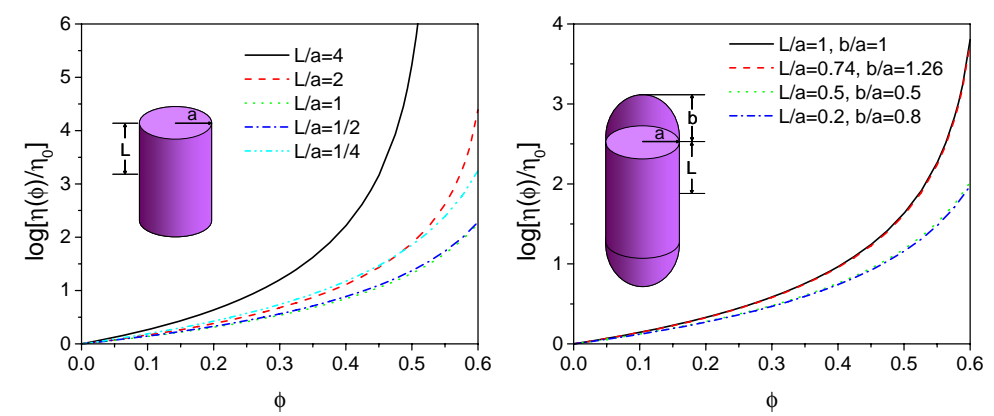

Fig. 4. Left panel: Relative viscosity $\eta(\phi) / \eta_{0}$ as predicted by our model for flat-ended cylinders with different aspect ratios. Right panel: The same as left panel but for hemiellipsoidal-ended cylinders with different aspect ratios. In both cases we assumed that the critical packing coincides with the random packing of spherocylinders with the same aspect ratio [54].

\subsection{Ellipsoids}

Another case in which the behavior of highly concentrated suspensions is of importance corresponds to suspensions of ellipsoidal particles, since they have been widely used as models of globular proteins and also because the hydrodynamic properties of ellipsoids are analytically known. For example, the intrinsic viscosity of an ellipsoid is given by [50]

$$
\begin{aligned}
{[\eta] } & =\frac{2}{5}\left(p^{2}-1\right)^{2}\left\{\frac{-\left(4 p^{2}-1\right) B+2 p^{2}+1}{3 p^{2}\left(3 B+2 p^{2}-5\right)\left[\left(2 p^{2}+1\right) B-3\right]}\right. \\
& +\frac{14}{3 p^{2}\left(3 B+2 p^{2}-5\right)}+\frac{2}{\left(p^{2}+1\right)\left(-3 p^{2} B+p^{2}+2\right)} \\
& \left.+\frac{p^{2}-1}{p^{2}\left(p^{2}+1\right)\left[\left(2 p^{2}-1\right) B-1\right]}\right\},
\end{aligned}
$$


Table 1

\begin{tabular}{|c|c|c|c|c|c|}
\hline$L / a$ & $1 / 4$ & $1 / 2$ & 1 & 2 & 4 \\
\hline$[\eta]$ & 3.92 & 3.05 & 2.87 & 3.40 & 5.27 \\
\hline$\phi_{c}(\approx)$ & 0.67 & 0.69 & 0.68 & 0.62 & 0.53 \\
\hline
\end{tabular}

Intrinsic viscosity $[\eta]$ [taken from Ref. ([8])] and critical packing $\phi_{c}$ [taken from Ref. $([54)]$ of flat-ended circular cylinders.

where

$$
\begin{aligned}
& B=p^{-1}\left(p^{2}-1\right)^{-1 / 2} \cosh ^{-1} p, \quad \text { when } \quad p>1, \\
& =1, \quad \text { when } \quad p=1 \text {, } \\
& =p^{-1}\left(1-p^{2}\right)^{-1 / 2} \cos ^{-1} p, \quad \text { when } \quad p<1 \text {. }
\end{aligned}
$$

Here, the axis ratio is defined by $p=b / a$ with $b$ the polar radius and $a$ is the equatorial radius. In the left panel of Fig. 3 we show the behavior of the intrinsic viscosity as a function of the axis ratio $p$. As can be seen, the lower value of the intrinsic viscosity occurs for spherical particles and increases slightly more sharply for prolate ellipsoids than for oblate ones. In the right panel of Fig. 3 we represent the viscosity as a function of the concentration $\phi$ for ellipsoids with different values of $p$. In all cases we have assumed that the critical packing coincides with the so called maximally random jammed (MRJ) state which corresponds to the least ordered among all jammed packings [51]. The critical densities of simulated packings of ellipsoids are calculated in Refs. ([52]) and ([53]) for different aspect ratios and we used them in the results of Fig. 3. As can be seen, the nonmonotonic behavior of the intrinsic viscosity shown in the left panel of Fig. 3 is reflected in the right panel of this figure.

\subsection{Cylinders}

Cylinders may be used to represent DNA molecules in certain physical conditions and also as models for rod-shaped viruses. Flat cylinders are encountered most often. Except in certain limit, analytical results are not available for hydrodynamic properties 
Table 2

\begin{tabular}{|c|c|c|c|c|}
\hline$L / a$ & 1 & 0.74 & 0.5 & 0.2 \\
\hline$b / a$ & 1 & 1.26 & 0.5 & 0.8 \\
\hline$[\eta]$ & 2.944 & 2.910 & 2.571 & 2.512 \\
\hline$\phi_{c}(\approx)$ & 0.62 & 0.62 & 0.68 & 0.68 \\
\hline
\end{tabular}

Intrinsic viscosity $[\eta]$ [taken from Ref. ([8])] and critical packing $\phi_{c}$ [taken from Ref. $([54])]$ of hemiellipsoidal-ended circular cylinders.

of cylinders. Thus, numerical calculations have to be used. In the limit that the total length $(2 L)$ goes to zero, a flat-ended cylinder becomes a disk. The intrinsic viscosity of a disk with radius $a$ can be found from Eq. (35) by taking the $p \rightarrow 0$ limit. The result is $[\eta]=128 a^{3} / 45$. In the case of globular cylinders for which the aspect ratio $L / a$ ranges from $1 / 4$ to 4 the hydrodynamic properties obtained numerically are listed in Table 1, [8]. Together with the hydrodynamic properties, in the last column of Table 1, we show the critical packing which we assume to coincide with the random packing of the cylinders. Actually, we are not aware of data for the close packing of flat-ended cylinders so that we are using the results for spherocylinders obtained in Ref. ([54]) with the same aspect ratio. The viscosity as a function of concentration is shown in Fig. 4.

In the case of circular cylinders with hemiellipsoidal ends, the results for the intrinsic viscosity [8] and the critical packing are listed in Table 2. As in the previous case, the value of the random packing listed corresponds to spherocylinders with the same aspect ratio defined as $(b+L) / a$ (see inset of Fig. 5$)$. The viscosity in this case is shown in Fig. 5 where one can see that it is larger for the cylinders with the largest aspect ratio.

Experimental data for the concentration and particle size-dependence of the low-shear viscosity of isotropic rod-dispersions are discussed in Ref. [9]. Four systems of stiff rods are considered: colloidal silicaboehmite, xanthan $(\lambda=0.5)$, schizophyllan $(\lambda=0.3)$ and non-Brownian PMMA-fibre. Here, $\lambda$ is the ratio of the polymer length and the persistence length. The measured intrinsic vis- 
Table 3

\begin{tabular}{|c|c|c|c|c|}
\hline & Silica rods & Schizophylian & Xanthan & PMMA-fibre \\
\hline$[\eta]$ & 50.2 & 42.7 & 58.1 & 27.6 \\
\hline$L / a$ & 44.0 & 46.0 & 56.0 & 39.8 \\
\hline$\phi_{c}(\approx)$ & 0.245 & 0.235 & 0.193 & 0.271 \\
\hline
\end{tabular}

Table 3. Intrinsic viscosity $[\eta]$ [taken from Ref. ([9])], aspect ratio $L / a$, and critical packing $\phi_{c}$ [obtained from Eq. 37]] for isotropic dispersions of rods.

cosities $[\eta]$ of these systems are tabulated in Table 3. The packing density is determined by their aspect ratio. For a random packing of thin hard rods, it was shown[55], [56] that

$$
\phi_{\max } \frac{L}{a}=\xi \quad \text { for } \frac{L}{a}>>1,
$$

where $\xi$ is the average number of contacts experienced by a rod. Experiments [55], [57] on random rod packing yield $\xi / 2=5.4$. This isotropic maximum packing fraction is a metastable glass with respect to the thermodynamically more favorable nematic phase as predicted by Onsager. The aspect ratio and the corresponding maximum packing calculated using Eq.(37) for the systems considered in Ref. [9], are also tabulated in Table 3. In Fig.5a we compare these data to our model using as parameters the values given in Table 3. As can be seen, poor agreement is found between the model and the experiment. A number of reasons can be argued to explain this result. For example, the silica rods are weakly attractive which implies a steeper viscosityconcentration curve as compared to the rigid macromolecules xanthan and schizophyllan. Of these two rod-like macromolecules the xanthan chain possesses higher flexibility which may also influence the viscosity data. Additionally, since long rod-shaped molecules "entangle" in the semidilute regime, it is expected that some orientational correlation arises at large concentrations and our model do not consider these effects.

To explore the possibility that the rods present some amount of orientational correlations, we have compared our model to the experimental data but considering that some alignment due to the 

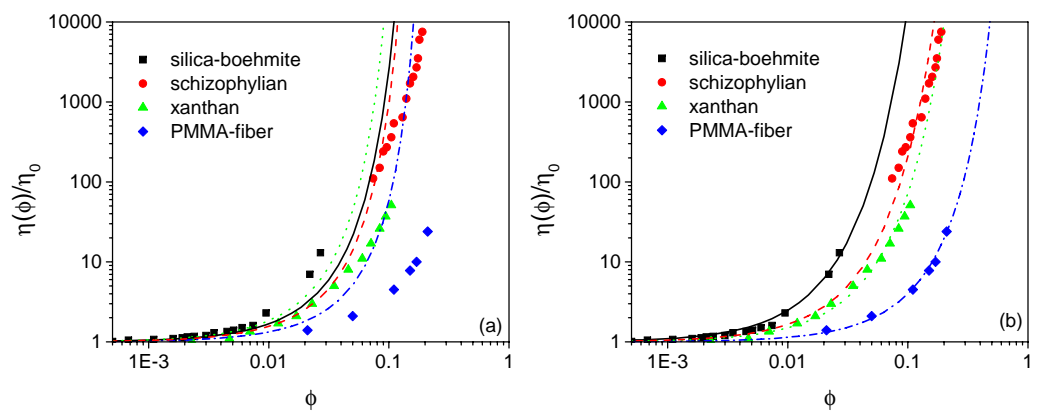

Fig. 5. Left panel: Relative viscosity $\eta(\phi) / \eta_{0}$ as predicted by our model for isotropic rods with different aspect ratios and intrinsic viscosities as given by Table 3. Silica rods (solid line), schizophylian (dashed line), xanthan (dotted line), and PMMA fibre (dash-dotted line). Right panel: The same as left panel but assuming orientational order that leads to $\phi_{\max } \simeq 0.9069$. The fitted values of $[\eta]$ were 90 for silica rods (solid line), 50 for schizophylian (dashed line), 40 for xanthan (dotted line), and 13 for PMMA fibre (dash-dotted line). The data sets were taken from Ref. 9 .

flow and the correlations is possible. In this case, the maximum packing is no longer given by Eq. (37) and we assume the opposite limit, that is, that the rods are completely aligned. Then the maximum packing takes the value corresponding to an hexagonal arrangement of parallel rods $\phi_{\max } \simeq 0.9069$. Then, the intrinsic viscosities for random rods as tabulated in Table 3 are no longer useful and we take $[\eta]$ as a fitting parameter. This comparison is shown in in Fig. 5b. As can be seen, a much better agreement is obtained using these assumptions. Thus, some degree of alignment is suggested by the model. Nonetheless, for a concentration dependent orientational correlation, the intrinsic viscosity $[\eta]$ is rather a function of $\phi$ and therefore, the values predicted by the present model may not be correct. 


\subsection{Dumbbells}

A dumbbell that consists of two spheres can be used as a model for a protein dimer or a protein consisting of two separate domains. Wakiya [7] and Brenner [6] calculated the intrinsic viscosity for dumbbells consisting of two equal-radius spheres at various separations. Here, we will only discuss the case when the ratio $L / a=1$, where $a$ is the radius of the spheres and $L$ is half of their center-to-center separation. In this case, $[\eta]=3.4496$.

The viscosity of dumbbells made of two fused spheres is shown in Fig. 6 and compared with the numerical results reported by in't Veld and coworkers for nanodimers [58]. The closed circles represent points assuming an effective volume fraction for particle radii adjusted to the peak onset in the nanoparticle pair distribution function. This adjustment corrects for the solvation shell around the nanoparticles when the solvent is treated explicitly [58]. The fitting parameter in this case was $\phi_{c} \simeq 0.58$, which is close to the critical packing value for the glass transition predicted by mode coupling theory $\phi_{c} \simeq 0.56$ (see Ref. [59]).

\subsection{Other Shapes}

Notice that the intrinsic viscosity $[\eta]$ for long rod-shaped particles takes values much larger as compared to the corresponding ones for spheres. However, it is possible to have very large values of the intrinsic viscosity without making a very extended or flat object [32]. An strategy to increase $[\eta]$ is to consider irregularly shaped particles like sponges or jack-like objects. The intrinsic viscosities for these shapes have been calculated numerically by finite element computations in Ref.[32]. We use these values in Fig. 7 to compare the viscosity-concentration curves for three representative cases: a sponge, a wire frame, a square ring, and a jack-like object. 


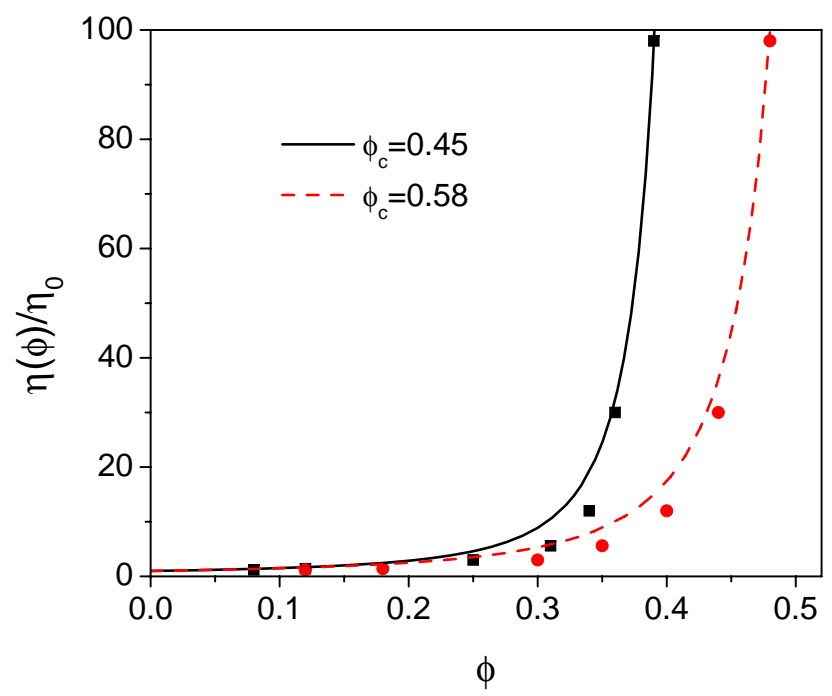

Fig. 6. Relative viscosity $\eta(\phi) / \eta_{0}$ as predicted by our model for a system of dumbbells. The closed squares represent points assuming radii of the spheres $a=5 \sigma$, where $\sigma$ is the size of a Lennard-Jones solvent atom in determining $\phi$, whereas the circles denote a volume fraction for radii adjusted to the peak onset in the nanoparticle pair distribution function.

The sponge is constructed starting with a cube in which a square channel is cut through the center of each face, which passes completely through the cube, as seen in Fig. 7. The parameter $m$ is taken to be the edge length of the cutout face in units of the cube edge length. When $m$ approaches 1 a rigid cubic wire frame is obtained. A similar procedure is employed to construct the flat square. The jack is constructed by poking three rectangular parallelepipeds orthogonally through a sphere. In all the curves of Fig. 7 we assumed $\phi_{c} \simeq 0.637$, the random close packing for spheres and the values of the intrinsic viscosity given in Ref.[32]. As expected, the objects with larger intrinsic viscosities have steeper curves. These examples are intended for illustrative purposes only, since we are unaware of experimental results to compare with. 


\section{Conclusions}

We have presented a simple model based on an effective-medium theory for the calculation of the viscosity of suspensions of arbitrarilyshaped particles as a function of particle concentration. The model considers excluded volume interactions between the particles through an effective filling fraction $\phi_{\text {eff }}$. This quantity introduces a universal scaling that may be used to reduce both experimental and theoretical results to a master curve [39], [40] which is independent of the experimental details or the shape of the particles.

Starting from known values and formulas for the intrinsic viscosity of the particles, the procedure yields to analytical expressions that predict the viscosity of the system for the whole range of concentrations. At low filling fractions it reduces to the correct limit while at high concentrations it diverges in a way similar to that predicted by mode coupling theories. In contrast to other models [30], our proposal contains only one fitting parameter which corresponds to the critical packing where the suspension loses its fluidity.

When applied to a suspension of spherical particles, our model improves considerably the predictions obtained using the well known Krieger and Dougherty model and any other model tested in the whole concentration range. We have employed our model to predict the viscosity of elliptical, and cylindrical particles, as well as dumbbells made of fused spheres and other complex shapes. In all cases where numerical or experimental data are available, the agreement with the proposed model is very good. It is convenient to emphasize that our model is not intended to describe correctly suspensions of large fibres, since in this case orientational correlations may exist. These correlations could introduce dependences of the intrinsic viscosities on the concentration. In a previous work [40] we have applied the procedure to emulsions of spherical droplets with equally good results. 


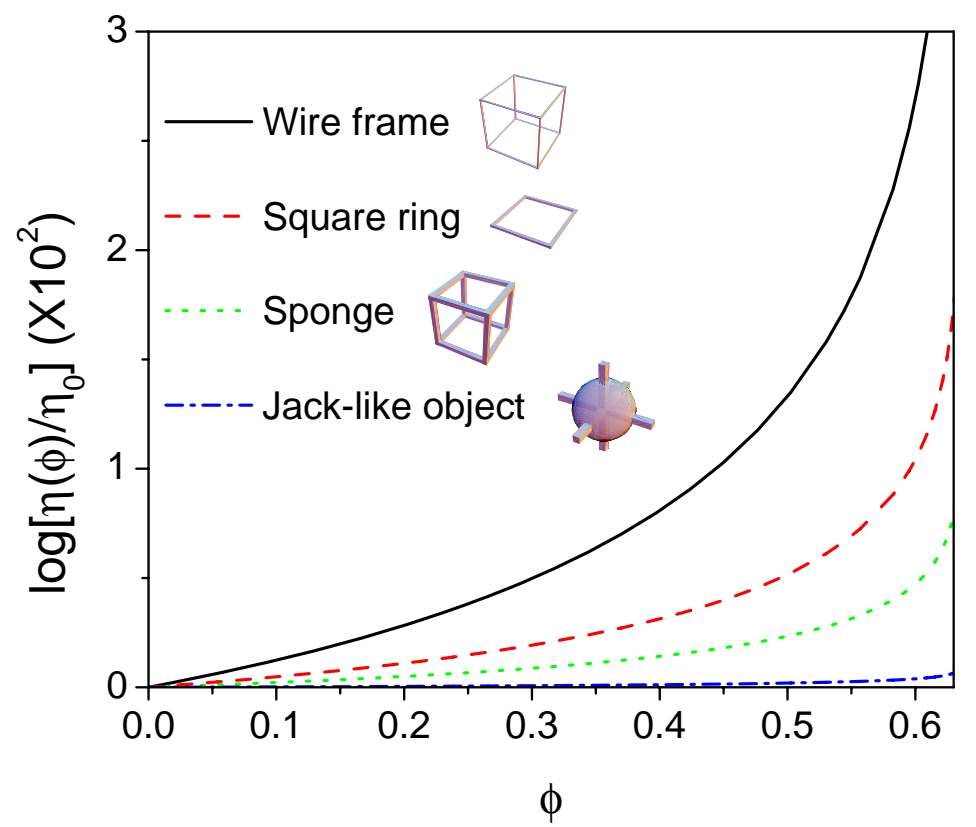

Fig. 7. Relative viscosity $\eta(\phi) / \eta_{0}$ as predicted by our model for a sponge $(m=23 / 27,[\eta]=44.7)$, a cubic wire frame $(m=33 / 35,[\eta]=255)$ a square ring $(m=23 / 25,[\eta]=98.7)$, and a jack-like object $([\eta]=3.68)$. The specific proportions of the jack are as follows, if the width of the parallelipipeds have unit length, the length has 15 units and the sphere diameter has 9 . In all cases we assumed $\phi_{c} \simeq 0.637$.

Due to the importance of shape effects on the rheological behavior of colloidal dispersions and despite that there are numerous reports for industrial systems but fewer data for suspensions with controlled geometry, we consider that the results presented in this article can help to provide a valuable characterization of these systems with very promising practical applications.

\section{Acknowledgements}

We thank Profs. G. S. Grest, M. K. Petersen, and P.J. in 't Veld for kindly sharing with us their numerical results for a suspension of dumbbells. This work was supported in part by Grants DGAPA IN-115010 (CIM) and IN-102609 (ISH). 


\section{References}

[1] A. Einstein, Investigations on the Theory of Brownian Movement (Dover, New York, 1975); Ann. Phys. 34, 591 (1911).

[2] G.B. Jeffery, Proc. Roy. Soc.,A 102,. 715 (1922).

[3] G.I.Taylor, Proc. R. Soc. London, Ser. A 138 (1932) 41-48.

[4] J.M. Rallison, J. Fluid Mechanics 84, 237 (1978).

[5] S. Haber and H. Brenner, J. Colloid Interface Sci. 97, 496 (1984).

[6] H. Brenner, Int. J. Multiphase Flow. 1, 195 (1974).

[7] S. Wakiya, J. Phys. Soc. Jap. 31, 1581 (1971).

[8] H-X Zhou, Biophys. J. 69, 2286 (1995).

[9] A.M. Wierenga and A.P. Philipse, Colloids and Surfaces A 137, 355 (1998).

[10] T.G.M. van de Ven, Colloidal Hydrodynamics (Academic Press, London, 1989).

[11] R.G. Larson, The Structure and Rheology of Complex Fluids (Oxford University Press, New York, 1999).

[12] J. Happel and H. Brenner, Low Reynolds number hydrodynamics (Kluwer, Dordrecht, 1991).

[13] M. Doi and S.F. Edwards, The Theory of Polymer Dynamics (Oxford University Press, New York, 1988).

[14] N. Saito, J. Phys. Soc. Japan 5, 4 (1950).

[15] J. M. Peterson and M. Fixman, J. Chem. Phys. 39, 2516 (1963).

[16] R. A. Lionberger and W. B. Russel, Adv. Chem. Phys. 111, 399 (2000).

[17] G. K. Batchelor and J. T. Green, J. Fluid Mech. 56, 401 (1972).

[18] G. K. Batchelor, J. Fluid Mech. 83, 97 (1977).

[19] P. Mazur and D. Bedeaux, Physica 76, 235 (1974).

[20] B. Cichocki, B.U. Felderhof, Phys Rev. A 46, 7723 (1992).

[21] B. Cichocki and B. U. Felderhof, Phys. Rev. A 43, 5405 (1991)

[22] R. Verberg, I.M. de Scheper, and E.G.D. Cohen, Phys. Rev. E 55, 3143 (1997).

[23] D. Bedeaux, J. Colloid Interface Sci. 118, 80 (1987).

[24] J. Smeets, et al, Langmuir 10, 1387 (1994).

[25] D. Bedeaux, R. Kapral, and P. Mazur, Physica 88A, 88 (1977). 
[26] J.C. van der Werff and C.G. de Kruiff, J. Rheol. 33, 421 (1989).

[27] C.W.J. Beenakker, Physica 128A, 48 (1984).

[28] G.K. Batchelor and J.T. Green, J. Fluid Mech. 56, 401 (1972).

[29] W.B. Russel and A.P. Gast, J. Chem. Phys. 84, 1815 (1986).

[30] J. Bicerano, J.F. Douglas, and D.A. Brune, J. Macromol. Chem. Phys. C39, 561 (1999).

[31] J.F. Brady, J. Chem. Phys. 99, 567 (1993).

[32] J.F. Douglas and E.J. Garboczi, Advances in Chemical Physics 91, 85 (1995).

[33] D. Quemada, Rheol. Acta 16, 82 (1977).

[34] H.C. Brinkman, J. Chem. Phys. 20, 571 (1952).

[35] R. Roscoe, Br. J. Appl. Phys. 3, 267 (1952).

[36] I.M. Krieger and T.J. Dougherty, Trans. Soc. Rheol. 3, 137 (1959).

[37] R. Pal and E. Rhodes, J. Rheol. 33, 1021 (1989).

[38] M. Mooney, J. Colloid Sci. 6, 162 (1951).

[39] C. I. Mendoza, I. Santamaría-Holek, J. Chem. Phys. 130, 044904 (2009).

[40] C. I. Mendoza, I. Santamaría-Holek, Appl. Rheol. 20, 23493 (2010).

[41] J. Mewis, N. J. Wagner, J. Non-Newtonian Fluid Mech. 157, 147 (2009).

[42] J.W. Bullard, A.T. Pauli, E.J. Garboczi, N.S. Martys, J. Colloid Interface Sci. 330, 186 (2009).

[43] V. Pryamitsyn and V. Ganesan, J. Chem. Phys. 122, 104906 (2005).

[44] Z. Cheng, et al, Phys. Rev. E 65, 041405 (2002).

[45] H.J.H. Clercx and P.P.J.M. Schram, Phys. Rev. A 45, 860 (1992).

[46] C. G. de Kruif, E. M. F. van Iersel, A. Vrij, and W. B. Russel, J. Chem. Phys. 83, 4717 (1986).

[47] I.M. Krieger, Advan. Colloid Interface Sci. 3, 111 (1972).

[48] J.C. van der Werff, C.G. de Kruiff, C. Blom, J. Mellema, Phys. Rev. A 39, 795 (1989).

[49] J.X. Zhu, D.J. Durian, J. Muller, D.A. Weitz, and D.J. Pine, Phys. Rev. Lett. 68, 2559 (1992).

[50] L.D. Landau, E.M. Lifshitz, and L.P. Pitaevskii, Electrodynamics of Continuous Media (Pergamon Press, Oxford 1984). 
[51] S. Torquato, T.M. Truskett, and P.G. Debenedetti, Phys. Rev. Lett. 84, 2064 (2000).

[52] A. Donev, et al, Science 303, 990 (2004).

[53] A. Donev, et al, Phys. Rev. E 75, 051304 (2007).

[54] S.R. Williams and A.P. Philipse, Phys. Rev. E 67, 051301 (2003).

[55] A.P. Philipse, Langmuir 12, 1127 (1996); Corrigendum: Langmuir 12, 5971 (1996).

[56] A.P. Philipse and A. Verberkmoes, Physica A 235, 186 (1997).

[57] M. Nardin, E. Papirer, and J. Schultz, J. Powder Technol. 44, 131 (1985).

[58] P.J. in't Veld, M.K. Petersen, and G.S. Grest, Phys. Rev. E 79, 021401 (2009).

[59] S.-H. Chong and W. Götze, Phys. Rev. E 65, 041503 (2002). 